\title{
Symmetry analysis of trimers rovibrational spectra: the case of $\mathrm{Ne}_{3}$
}

\author{
Maykel Márquez-Mijares ${ }^{1}$, Octavio Roncero ${ }^{2}$, Pablo Villarreal $^{2}$, and Tomás González-Lezana ${ }^{2}$ \\ 1 Instituto Superior de Tecnologías y Ciencias Aplicadas, Ave. Salvador Allende y Luaces, Quinta de los Molinos, Plaza, 10600 \\ La Habana (Cuba) \\ 2 Instituto de Física Fundamental, Consejo Superior de Investigaciones Científicas, IFF-CSIC, Serrano 123 , 28006 Madrid \\ (Spain)
}

Received: date / Revised version: date

\begin{abstract}
An approximate method to assign the symmetry to the rovibrational spectrum of homonuclear treatments based on the solution of the rotational Hamiltonian by means of a purely vibrational basis combined with standard rotational functions is applied on $\mathrm{Ne}_{3}$. The neon trimer constitutes an ideal test between heavier systems such as $\mathrm{Ar}_{3}$ for which the method proves to be an extremely useful technique and some other previously investigated cases such as $\mathrm{H}_{3}^{+}$where some limitations were observed. Comparisons of the calculated rovibrational energy levels are established with results from different calculations reported in the literature.
\end{abstract}

PACS. PACS-key discribing text of that key - PACS-key discribing text of that key

\section{Introduction}

Rare gas ( $\mathrm{RG}$ ) trimers have been the subject of a large list of works focussed in the study of their bound states energies and structures [1-10]. Theoretical approaches developed to characterize the molecular rotation-vibration structure have been currently tested on these kind of systems [11-16]. Among all these numerous investigations we proposed some time ago a method to calculate and assign the corresponding symmetry character to the rovibrational spectra of $\mathrm{Ar}_{3}$ for different values of the total angular momentum $J>0[17,18]$. The approach was based in a previously developed method to solve the pure vibrational problem, $J=0$, using a basis set constructed with distributed Gaussian functions (DGFs) on the interparticle distances [19]. This method for the rotationless case was successfully applied for different RG trimers [2023 ], and subsequently extended to treat $J>0$ situations [24-26]. In the same spirit of a comparative analysis performed before between different $\mathrm{RG}$ trimers [5,21] we test in this work the DGF rotation-vibration approach developed in Ref. [17] with $\mathrm{Ne}_{3}$. The difference with mass in comparison with the Ar trimer, an example for which the performance of the presently discussed method was found remarkably good $[17,18]$, is expected to constitute a more demanding challenge. In fact, a previous application [27] on a much lighter system such as $\mathrm{H}_{3}^{+}$revealed some limitations due to the importance of the contribution from

$\overline{\text { Send offprint requests to: }}$
Coriolis distortion terms which the present approximative approach does not explicitly account for.

The rotational spectrum of $\mathrm{Ne}_{3}$ has been investigated before by different authors. For example, Salci et al. [13] employed a finite elements method (FEM) to calculate rovibrational states up to $J=3$ assuming the trimer as an oblate symmetric top. The comparison with the analytical expression of the energy levels corresponding to a symmetric planar trimer revealed increasing differences with the vibrational excitation. Moreover significant contribution from Coriolis coupling terms was observed. The hyperspherical coordinates (HC) calculation by Suno et al. [15] employed the same pairwise potential than Ref. [13] and reported results up to $J=6$. Finally, energy levels and wave functions corresponding to the neon trimer bound states were obtained in quantum dynamical calculations by Yang et al. [14] between $J=0-18$ using a Lennard-Jones potential. In this work we compare with some of these most recent studies on the title system to test the capabilities of the DGF method to treat the rotation-vibration spectrum of these $R G$ trimers.

The paper is structured as follows: Theoretical details of the DGF method are given in Section 2; results are discussed in Section 3 with a separate comparison with results from Refs. [13] and [15], and finally, conclusions are shown in Section 4. 


\section{Theory}

The method proposed in previous investigations of $\mathrm{Ar}_{3}$ $[17,18]$ assumes the total Hamiltonian as the sum of a vibrational and a rotational part: $H_{T}=H_{V}+H_{R}$. The expression of $H_{V}$ in terms of interparticle distances $R_{i}$ for the case of three identical atoms with mass $m_{i}$, can be finally expressed, after transformations of the total wave function $[20,24]$, as follows:

$$
H_{V}=\sum_{i=1}^{3}\left\{\frac{-\hbar^{2}}{m_{i}}\left[\frac{\partial^{2}}{\partial R_{i}^{2}}+\frac{1}{R_{i}}+\frac{\partial}{\partial R_{i}}-\frac{1}{4 R_{i}^{2}}+T_{i j k}\right]\right\}+U(\mathbf{R})
$$

where $U(\mathbf{R})$ is the potential energy operator and the $T_{i j k}$ operator, with $i \neq j \neq k$, is written as:

$$
T_{i j k}=\cos \theta_{i}\left[\nabla_{j} \nabla_{k}-\frac{\nabla_{k}}{2 R_{j}}-\frac{\nabla_{j}}{2 R_{k}}+\frac{1}{4 R_{j} R_{k}}\right]
$$

where $\nabla_{i}=\partial / \partial R_{i}$ and

$$
\cos \theta_{i}=\frac{R_{j}^{2}+R_{k}^{2}-R_{i}^{2}}{2 R_{j} R_{k}}
$$
30]:

For $H_{R}$, we choose the case of an asymmetric top [28-

$$
H_{R}=\frac{1}{2}(A+C) \mathbf{J}^{2}+\frac{1}{2}(A-C)\left(J_{A}^{2}+\kappa J_{B}^{2}-J_{C}^{2}\right)
$$

where $\kappa=(2 B-A-C) /(A-C)$ is Ray's asymmetry parameter [31] and $A, B$ and $C$ are rotational constants.

In order to solve the vibrational part, we employ symmetry adapted basis functions for each irreducible representation corresponding to the $C_{3 v}$ group for homonuclear trimers as follows:

$$
\begin{aligned}
\phi_{j}(\mathbf{R}) & =\frac{1}{\sqrt{6}}\left\{\chi(E) \mathcal{G}_{l m n}^{123}(\mathbf{R})+\chi\left(C_{3}\right)\left[\mathcal{G}_{l m n}^{231}(\mathbf{R})+\mathcal{G}_{l m n}^{312}(\mathbf{R})\right]\right. \\
& \left.+\quad \chi\left(\sigma_{v}\right)\left[\mathcal{G}_{l m n}^{213}(\mathbf{R})+\mathcal{G}_{l m n}^{132}(\mathbf{R})+\mathcal{G}_{l m n}^{312}(\mathbf{R})\right]\right\}
\end{aligned}
$$

where the $\mathcal{G}_{l m n}^{123}(\mathbf{R})$ are products of $\varphi_{p}\left(R_{i}\right)$ Gaussian functions for the $R_{i}$ distance with center at the value $R_{p}$ :

$$
\mathcal{G}_{l m n}^{123}(\mathbf{R})=\varphi_{l}\left(R_{1}\right) \varphi_{m}\left(R_{2}\right) \varphi_{n}\left(R_{3}\right)
$$

$\chi(E), \chi\left(C_{3}\right)$ and $\chi\left(\sigma_{v}\right)$ are the characters for the $C_{3 v}$ group, and once their corresponding values are introduced in Equation (5) for each irreducible representation, we obtain the following basis functions set for $A_{1}, A_{2}$ and $E$ as follows:

$$
\begin{aligned}
\phi_{j}^{A_{1}}(\mathbf{R})= & \frac{1}{\sqrt{6}}\left[\mathcal{G}_{l m n}^{123}(\mathbf{R})+\mathcal{G}_{l m n}^{231}(\mathbf{R})+\mathcal{G}_{l m n}^{312}(\mathbf{R})\right. \\
+ & \left.\mathcal{G}_{l m n}^{213}(\mathbf{R})+\mathcal{G}_{l m n}^{132}(\mathbf{R})+\mathcal{G}_{l m n}^{321}(\mathbf{R})\right] \\
\phi_{j}^{A_{2}}(\mathbf{R})= & \frac{1}{\sqrt{6}}\left[\mathcal{G}_{l m n}^{123}(\mathbf{R})+\mathcal{G}_{l m n}^{231}(\mathbf{R})+\mathcal{G}_{l m n}^{312}(\mathbf{R})\right. \\
& \left.-\mathcal{G}_{l m n}^{213}(\mathbf{R})-\mathcal{G}_{l m n}^{132}(\mathbf{R})-\mathcal{G}_{l m n}^{321}(\mathbf{R})\right] \\
\phi_{j}^{E}(\mathbf{R})= & \frac{1}{\sqrt{6}}\left[2 \mathcal{G}_{l m n}^{123}(\mathbf{R})-\mathcal{G}_{l m n}^{231}(\mathbf{R})-\mathcal{G}_{l m n}^{312}(\mathbf{R})\right]
\end{aligned}
$$

In combination with the eigenfunctions of $H_{V}$ for the $J=0$ case, we construct a basis set employing standard rotational basis functions in terms of $J$ and its projections on the $z$ axis of the body-fixed $(\Omega)$ and space-fixed $(M)$ frames to diagonalize $H_{R}$ from Equation (4). As a result of the above discussed approach to obtain the rovibrational spectrum, the symmetry of the total wave function for the corresponding bound states can be expressed as the product:

$$
\Gamma=\Gamma_{V}^{\Omega \ell} \times \Gamma_{R}
$$

where $\Gamma_{R}$ refers to the symmetry of the strictly rotational part, while $\Gamma_{V}^{\Omega \ell}$ refers to a rovibrational part, where $\ell$ is the vibrational angular momentum number [28]. In our previous studies $[17,18]$ we developed a recipe to assign the character for $\Gamma_{R}$ and $\Gamma_{V}^{\Omega \ell}$. In essence we can summarize as follows: If $\Omega=0, \Gamma_{R}=A_{1}\left(A_{2}\right)$ for even (odd) values of $J$, and $\Gamma_{\mathrm{R}}=A_{1}$ or $A_{2}$ for $\Omega \neq 0$. On the other hand, for the rovibrational part, if we define $\eta=| \pm 2 \ell-\Omega|$, with $\ell, \Omega \geq 0$, the rule consists on: (i) $\Gamma_{V}^{\Omega \ell}=A_{1}$ for $\eta=0$; (ii) $\Gamma_{V}^{\Omega \ell}=A_{1}, A_{2}$ for $\eta$ multiple of 3 ; and (iii) $\Gamma_{V}^{\Omega \ell}=E$ if $\eta$ is not multiple of 3 . Finally, and due to the fact that our basis set has been adapted to symmetry according to the $C_{3 v}$ group instead of $D_{3 h}$, the total symmetry $\Gamma$ in equation (10) is ' or " depending on whether $\Omega$ is even or odd, respectively.

In order to assign the quantum number $\ell$ to the vibrational states obtained with the DGF approach, the HC method from Ref. [18] has been used. The DGF calculation was performed using up to 50 Gaussian functions $\varphi_{i}(R)$ with centers starting from $2.38 \AA$ equally spaced with an interval of $0.16 \AA$. A total number of $19,053 \phi_{j}^{A_{1}}$ functions from Equation (7) were generated for the $A_{1}$ representation; $15,877 \phi_{j}^{A_{2}}$ functions from Equation (8) for the representation $A_{2}$ and, finally $36,790 \phi_{j}^{E}$ functions from Equation (9) for $E$. Details of how the matrix elements of the total Hamiltonian, which contains crossed terms between $\Omega$ and $\Omega \pm 2$, and are calculated in a DGF basis set have been given before and the interested reader is referred to those previous papers $[24,17,18]$. For the scope of this work, it will suffice by indicating that the integration in each $R_{i}$ coordinate involved in both $H_{V}$ and $H_{R}$ of Equations (1) and (4), respectively, is solved applying a mean-value approach for the centers of the DGF.

In this work present DGF results have been compared with those reported from the previous investigations on $\mathrm{Ne}_{3}$ by Salci et al. [13] and by Suno [15]. The potential energy operator $U(\mathbf{R})$ in Equation (1) employed in the calculations was the HFD-B pairwise potential by Aziz and Slaman [32].

\section{Results}

\subsection{Comparison with calculation by Salci et al. [13]}

The calculation by means of the FEM method by Salci et al. [13], as opposed to the study performed by Suno [15] 
Table 1. Energy levels of the $\mathrm{Ne}_{3}$ rovibrational states for $J=0$ (in $\mathrm{cm}^{-1}$ ) obtained in this work by means of $\mathrm{HC}$ (third column) and DGF (seventh column) methods. The $\ell$ number is given in the second column and the $v^{\Gamma}$ vibrational number for each irreducible representation is in the sixth column. Energies and the $v$ number for the vibrational states from Ref. [13] are given in the fourth and fifth columns, respectively.

\begin{tabular}{|c|c|c|c|c|c|c|}
\hline & $\mathrm{HC}$ & & FEM [13] & & DG & \\
\hline$\Gamma$ & $\ell$ & $E$ & $v$ & $E$ & $v^{\Gamma}$ & $E_{\mathrm{DGF}}$ \\
\hline \multirow[t]{9}{*}{$A_{1}^{\prime}$} & 0 & -51.51 & 0 & -51.50 & 1 & -51.51 \\
\hline & 0 & -36.44 & 2 & -36.43 & 2 & -36.44 \\
\hline & 3 & -34.22 & 4 & -34.22 & 3 & -34.35 \\
\hline & 0 & -31.64 & 6 & -31.63 & 4 & -31.85 \\
\hline & 0 & -28.04 & 8 & -28.04 & 5 & -28.22 \\
\hline & 0 & -24.09 & 10 & -24.08 & 6 & -24.14 \\
\hline & 0 & -22.46 & 12 & -22.45 & 7 & -22.46 \\
\hline & 0 & -21.91 & 14 & -21.90 & 8 & -22.06 \\
\hline & 0 & -19.22 & 18 & -19.21 & 9 & -19.17 \\
\hline \multirow[t]{9}{*}{$E^{\prime}$} & 1 & -38.76 & 1 & -38.75 & 1 & -38.76 \\
\hline & 1 & -34.63 & 3 & -34.63 & 2 & -34.71 \\
\hline & 2 & -32.42 & 5 & -32.42 & 3 & -32.63 \\
\hline & 2 & -28.65 & 7 & -28.64 & 4 & -28.87 \\
\hline & 1 & -24.55 & 9 & -24.54 & 5 & -24.57 \\
\hline & 1 & -23.46 & 11 & -23.45 & 6 & -23.48 \\
\hline & 1 & -22.36 & 13 & -22.34 & 7 & -22.44 \\
\hline & 1 & -20.23 & 15 & -20.23 & 8 & -20.26 \\
\hline & 1 & -19.65 & 17 & -19.65 & 9 & -19.62 \\
\hline \multirow[t]{2}{*}{$A_{2}^{\prime}$} & 3 & -20.06 & 16 & -20.05 & 1 & -20.06 \\
\hline & 3 & -18.50 & 19 & -18.49 & 2 & -18.60 \\
\hline
\end{tabular}

focussed only in the $A_{1}^{\prime}$ and $A_{1}^{\prime \prime}$ levels, comprised three different irreducible representations $\left(A_{1}^{\prime}, A_{2}^{\prime}\right.$ and $\left.E^{\prime}\right)$. We will therefore use the comparison with the former results to detail some of the aspects of the symmetry assignment procedure of the DGF method.

One of the first steps consists on the assignment of the $\ell$ number to the $H_{V}$ eigenstates obtained with the DGF approach required for the symmetry characterization of the rotational spectra. This is usually achieved by simply comparison fo the $J=0$ energy levels with the results obtained by means of a $\mathrm{HC}$ calculation. Table 1 shows vibrational levels obtained in this work with $\mathrm{HC}$ and DGF methods in comparison with results reported in Ref. [13]. The energy values of the $A_{1}^{\prime}$ levels from Ref. [18] are in perfect agreement with the present $\mathrm{HC}$ calculation, which extends, as shown in Table 1 , to the $E^{\prime}$ and $A_{2}^{\prime}$ groups. The diagonalization of the rotationless Hamiltonian of Equation (1) in separate symmetry-adapted basis functions as shown in Equations (7)-(9) reveals that even values of the index $v$ in the work by Salci et al. [13] corresponds to the $A_{1}^{\prime}$ representation whereas odd values are for the $E^{\prime}$ block. The only exception to this were the lowest states of the $A_{2}^{\prime}$ irreducible representation which appears below $\sim-20$ $\mathrm{cm}^{-1}$. On the other hand the $A_{1}^{\prime}$ block is the only one containing states with $\ell=0$.
Table 2. Comparison between energy levels of the $\mathrm{Ne}_{3}$ rovibrational states for $J=1$, measured in $\mathrm{cm}^{-1}$ obtained by means of the FEM method of Ref. [13] (third column) and the present DGF (ninth column).

\begin{tabular}{lllllllll}
\hline$\Gamma_{\mathrm{FEM}}$ & $\Omega$ & $E_{\mathrm{FEM}}$ & $\Gamma_{V}^{\Omega \ell}$ & $\Gamma_{R}$ & $\Gamma$ & $v^{\Gamma}$ & $\ell$ & $E_{\mathrm{DGF}}$ \\
\hline \multirow{4}{*}{$A$} & 0 & -51.18 & $A_{1}$ & $A_{2}$ & $A_{2}^{\prime}$ & $1^{A_{1}}$ & 0 & -51.19 \\
& 1 & -38.49 & $A_{1} / A_{2}$ & $A_{1} / A_{2}$ & $A_{2}^{\prime \prime}$ & $1^{E}$ & 1 & -38.52 \\
& 1 & -38.40 & $A_{1} / A_{2}$ & $A_{1} / A_{2}$ & $A_{1}^{\prime \prime}$ & $1^{E}$ & 1 & -38.50 \\
& 0 & -36.08 & $A_{1}$ & $A_{2}$ & $A_{2}^{\prime}$ & $2^{A_{1}}$ & 0 & -36.08 \\
\hline \multirow{4}{*}{$E$} & & & & & & & & \\
& 1 & -51.26 & $E$ & $A_{1} / A_{2}$ & $E^{\prime \prime}$ & $1^{A_{1}}$ & 0 & -51.27 \\
& 1 & -38.59 & $E$ & $A_{1} / A_{2}$ & $E^{\prime \prime}$ & $1^{E}$ & 1 & -38.55 \\
& 0 & -38.43 & $E$ & $A_{2}$ & $E^{\prime}$ & $1^{E}$ & 1 & -38.45 \\
& 1 & -36.19 & $E$ & $A_{2}$ & $E^{\prime \prime}$ & $2^{A_{1}}$ & 0 & -36.18 \\
\hline
\end{tabular}

The comparison between the rovibrational states for $J=1$ obtained with the present DGF method and those reported in the FEM calculation [13] is shown in Table 2. The assignment of the symmetry allows a more detailed characterization into the different irreducible representation blocks. The ground vibrational $1^{E}$ is found to be responsible of states in each of these symmetry groups $E$, $A_{1}$ and $A_{2}$, with the ' and " character depending on the even/odd $\Omega$. In view of the restricted energy range considered by Salci et al. in their work, none of the $J=0$ levels from the $A_{2}^{\prime}$ group shown in Table 1 contributes to the $J=1$ spectrum considered in this calculation. In turn, both $1^{A_{1}}$ and $2^{A_{1}}$ vibrational states are responsible of, respectively, the ground and first excited $J=1$ levels of $A_{2}^{\prime}$ and $E^{\prime \prime}$. The agreement between the DGF and FEM calculations for the energy values is remarkable, thus suggesting the good capabilities of the former approach, at least for these low energy levels of the $J>0$ case.

A similar impression is obtained when the calculation extends to $J=3$. The comparison between results from the DGF approach and the FEM study reported in Ref. [13] shown in Table 3 reveals differences between the corresponding energy values which do not exceed $\sim 0.2 \mathrm{~cm}^{-1}$ in any case. Ground states in every irreducible representation come from the $1^{A_{1}}$ vibrational state from the $J=0$ calculation, with degenerate levels which separate between $A_{1}^{\prime \prime}$ and $A_{2}^{\prime \prime}$. The larger number of different possibilities for $\Omega$ makes the $J=3$ spectrum denser although, as dicussed before for the $J=1$ case, the only vibrational states involved are $1^{A_{1}}, 2^{A_{1}}$ and $1^{E}$. As shown in Table 3 the symmetry of all states from the $E$ group mainly comes from the rovibrational part . In all cases, the $\eta$ number (see Section 2) becomes non multiple of 3 and, therefore $\Gamma_{V}^{\Omega \ell}=E$, a feature which solves the uncertainty between $A_{1}$ and $A_{2}$ coming from the rotational part $\Gamma_{R}$.

\subsection{Comparison with calculation by Suno [15]}

The rovibrational states obtained in Ref. [15] corresponds exclusively to the $A$ group and the only distinction is considered as a function of the even/odd character of $\Omega$, thus 
Table 3. Same as Table 2 for $J=3$.

\begin{tabular}{cllllllll}
\hline$\Gamma$ & $\Omega$ & $E_{\mathrm{FEM}}$ & $\Gamma_{V}^{\Omega \ell}$ & $\Gamma_{R}$ & $\Gamma$ & $v^{\Gamma}$ & $\ell$ & $E_{\mathrm{DGF}}$ \\
\hline \multirow{4}{*}{$A$} & 3 & -50.33 & $A_{1} / A_{2}$ & $A_{1} / A_{2}$ & $A_{1}^{\prime \prime}$ & $1^{A_{1}}$ & 0 & -50.35 \\
& 3 & -50.33 & $A_{1} / A_{2}$ & $A_{1} / A_{2}$ & $A_{2}^{\prime \prime}$ & $1^{A_{1}}$ & 0 & -50.35 \\
& 0 & -49.61 & $A_{1}$ & $A_{2}$ & $A_{2}^{\prime}$ & $1^{A_{1}}$ & 0 & -49.62 \\
& 2 & -37.44 & $A_{1}$ & $A_{1} / A_{2}$ & $A_{1}^{\prime}$ & $1^{E}$ & 1 & -37.44 \\
& 2 & -37.39 & $A_{1}$ & $A_{1} / A_{2}$ & $A_{1}^{\prime \prime}$ & $1^{E}$ & 1 & -37.29 \\
& 1 & -37.20 & $A_{1} / A_{2}$ & $A_{1} / A_{2}$ & $A_{1}^{\prime \prime}$ & $1^{E}$ & 1 & -37.21 \\
& 1 & -36.67 & $A_{1} / A_{2}$ & $A_{1} / A_{2}$ & $A_{2}^{\prime \prime}$ & $1^{E}$ & 1 & -36.81 \\
& 3 & -35.38 & $A_{1} / A_{2}$ & $A_{1} / A_{2}$ & $A_{1}^{\prime \prime}$ & $2^{A_{1}}$ & 0 & -35.29 \\
& 0 & -34.53 & $A_{1}$ & $A_{2}$ & $A_{2}^{\prime}$ & $2^{A_{1}}$ & 0 & -34.35 \\
\hline & & & & & & & & \\
$E$ & 2 & -49.93 & $E$ & $A_{1} / A_{2}$ & $E^{\prime}$ & $1^{A_{1}}$ & 0 & -49.94 \\
& 1 & -49.69 & $E$ & $A_{1} / A_{2}$ & $E^{\prime}$ & $1^{A_{1}}$ & 0 & -49.70 \\
3 & -37.91 & $E$ & $A_{1} / A_{2}$ & $E^{\prime \prime}$ & $1^{E}$ & 1 & -37.65 \\
& 3 & -37.53 & $E$ & $A_{1} / A_{2}$ & $E^{\prime \prime}$ & $1^{E}$ & 1 & -37.63 \\
2 & -37.27 & $E$ & $A_{1} / A_{2}$ & $E^{\prime}$ & $1^{E}$ & 1 & -37.25 \\
1 & -37.02 & $E$ & $A_{1} / A_{2}$ & $E^{\prime \prime}$ & $1^{E}$ & 1 & -37.01 \\
& 0 & -36.75 & $E$ & $A_{1} / A_{2}$ & $E^{\prime}$ & $1^{E}$ & 1 & -36.85 \\
2 & -34.86 & $E$ & $A_{1} / A_{2}$ & $E^{\prime}$ & $2^{A_{1}}$ & 0 & -34.84 \\
1 & -34.66 & $E$ & $A_{1} / A_{2}$ & $E^{\prime \prime}$ & $2^{A_{1}}$ & 0 & -34.58 \\
& & & & & &
\end{tabular}

Table 4. Comparison between energy levels of the $\mathrm{Ne}_{3}$ rovibrational states for $J=1$, measured in $\mathrm{cm}^{-1}$ obtained in Ref. [15] (second column) and the present DGF calculation (seventh colum).

\begin{tabular}{ccccccc}
\hline$v$ & $E[15]$ & $\Gamma$ & $\Omega$ & $v^{\Gamma}$ & $\ell$ & $E_{\mathrm{DGF}}$ \\
\hline $0^{-}$ & -38.42 & $A_{1}^{\prime \prime}$ & 1 & $1^{E}$ & 1 & -38.50 \\
$1^{-}$ & -33.70 & & 1 & $2^{E}$ & 1 & -33.83 \\
$2^{-}$ & -30.60 & & 1 & $3^{E}$ & 1 & -31.14 \\
$3^{-}$ & -26.33 & & 1 & $4^{E}$ & 1 & -27.35 \\
$4^{-}$ & -24.12 & & 1 & $5^{E}$ & 1 & -24.13 \\
$5^{-}$ & -21.05 & & 1 & $6^{E}$ & 1 & -22.16 \\
$6^{-}$ & -20.86 & & 1 & $7^{E}$ & 1 & -21.16 \\
$7^{-}$ & -19.64 & & 1 & $8^{E}$ & 1 & -19.42 \\
$8^{-}$ & -18.64 & & 1 & $9^{E}$ & 1 & -18.69 \\
\hline $0^{+}$ & -19.69 & $A_{1}^{\prime}$ & 0 & $1^{A_{2}}$ & 3 & -19.72 \\
$1^{+}$ & -17.50 & & 0 & $2^{A_{2}}$ & 3 & -17.35 \\
\hline
\end{tabular}

leading to the $A_{1}^{\prime \prime}$ and $A_{1}^{\prime}$ cases respectively. In Reference [15] levels have been identified as $v^{ \pm}$to distinguish between both groups. Once the assignment of the $\ell$ number to the DGF states for $J=0$ has already been made before (see Table 1 ) we start directly with $J=1$. The comparison between present results and those obtained in the calculation by Suno directly for that value of the total angular momentum is shown in Table 4 . The $J=1$ spectrum, restricted in this case to the $A$ group, involves more vibrational states than the previous comparison with results by Salci et al. [13]. In particular, all the $J=0$ spectrum corresponding to the $E$ block shown in Table 1 for the rotationless case, is present as original seeds of the rovibrational levels of the $A_{1}^{\prime \prime}$ group in Table 4 . The accord
Table 5. Same as Table 4 for $J=4$.

\begin{tabular}{cccclcc}
\hline$v$ & $E[15]$ & $\Gamma$ & $\Omega$ & $v^{\Gamma}$ & $\ell$ & $E_{\mathrm{DGF}}$ \\
\hline $0^{-}$ & -49.08 & $A_{1}^{\prime \prime}$ & 3 & $1^{A_{1}}$ & 0 & -49.09 \\
$1^{-}$ & -36.22 & & 1 & $1^{E}$ & 1 & -35.70 \\
$2^{-}$ & -34.09 & & 3 & $2^{A_{1}}$ & 0 & -34.09 \\
$3^{-}$ & -32.24 & & 1 & $2^{E}$ & 1 & -32.23 \\
$4^{-}$ & -29.69 & & 3 & $3^{A_{1}}$ & 3 & -30.00 \\
$5^{-}$ & -28.86 & & 1 & $3^{E}$ & 1 & -29.45 \\
$6^{-}$ & -25.22 & & 3 & $5^{E}$ & 0 & -25.15 \\
\hline & & & & & & \\
$0^{+}$ & -48.36 & $A_{1}^{\prime}$ & 0 & $1^{A_{1}}$ & 0 & -48.37 \\
$1^{+}$ & -36.90 & & 4 & $1^{E}$ & 1 & -36.97 \\
$2^{+}$ & -36.21 & & 2 & $1^{E}$ & 1 & -36.04 \\
$3^{+}$ & -33.73 & & 0 & $2^{A_{1}}$ & 0 & -34.04 \\
$4^{+}$ & -33.10 & & 4 & $2^{E}$ & 1 & -31.83 \\
$5^{+}$ & -31.40 & & 4 & $2^{E}$ & 1 & -31.30 \\
$6^{+}$ & -30.83 & & 2 & $3^{E}$ & 1 & -30.23 \\
$7^{+}$ & -28.11 & & 0 & $3^{A_{1}}$ & 3 & -28.15 \\
\hline
\end{tabular}

between the values of the energies is also good although slightly larger differences are seen than in the comparison established in Table 2 with results from Ref. [13]. The onset of the $A_{1}^{\prime}$ branch, on the other hand, occurs at much higher energies, in fact the ground state of such symmetry block lies above seven states of $A_{1}^{\prime \prime}$, although in the calculation by Suno [15] the energy difference between such a $A_{1}^{\prime}$ ground state and the $7^{-}$state of the $A_{1}^{\prime \prime}$ block is smaller than the difference found in our study.

Calculations for larger values of the total angular momentum have been performed using the DGF approach. The comparison with results obtained in the work by Suno [15] for $J=4$ and 5 is presented in Tables 5 and 6 , respectively. The agreement between both set of energies is certainly good for almost the entire spectrum under consideration. The increase in the values of $\Omega$ as $J$ becomes larger does not constitute, for the symmetry blocks here investigated, any difficulty for the method.

Although we do not show it here (a different potential energy surface and even mass for the Ne atom are considered with respect to the previously discussed calculations) the comparison with values reported in a similar investigation of the Ne trimer rotational spectrum by Yang [14] also support the validity of the present DGF method. In fact, despite the lighter mass, $\mathrm{Ne}_{3}$ constitutes, as $\mathrm{Ar}_{3}[17,18]$, a good test to prove the capabilities of the approach both to calculate the rovibrational states of molecular trimers and to characterize the symmetry of such levels.

\section{Conclusions}

A previously proposed method based in the use of distributed Gaussian functions (DGF) to calculate and characterize the rovibrational spectrum of trimers has been employed for the case of $\mathrm{Ne}_{3}$. The comparison with previous investigations of the spectra for this system has revealed that the DGF method allows the calculation of the 
Table 6. Same as Table 5 for $J=5$.

\begin{tabular}{lllllll}
\hline$v$ & $E[15]$ & $\Gamma$ & $\Omega$ & $v^{\Gamma}$ & $\ell$ & $E_{\mathrm{DGF}}$ \\
\hline $0^{-}$ & -47.52 & $A_{1}^{\prime \prime}$ & 3 & $1^{A_{1}}$ & 0 & -47.52 \\
$1^{-}$ & -36.73 & & 5 & $1^{E}$ & 1 & -36.17 \\
$2^{-}$ & -33.81 & & 1 & $1^{E}$ & 1 & -33.77 \\
$3^{-}$ & -32.60 & & 3 & $2^{A_{1}}$ & 0 & -32.63 \\
$4^{-}$ & -31.87 & & 1 & $2^{E}$ & 1 & -31.47 \\
$5^{-}$ & -29.31 & & 5 & $3^{E}$ & 1 & -29.86 \\
$6^{-}$ & -28.38 & & 3 & $3^{A_{1}}$ & 3 & -28.11 \\
$7^{-}$ & -25.87 & & 3 & $4^{A_{1}}$ & 3 & -25.86 \\
$8^{-}$ & -24.86 & & 3 & $5^{A_{1}}$ & 0 & -24.57 \\
\hline & & & & & & \\
$0^{+}$ & -35.04 & $A_{1}^{\prime}$ & 4 & $1^{E}$ & 0 & -35.47 \\
$1^{+}$ & -34.19 & & 2 & $1^{E}$ & 1 & -34.60 \\
$2^{+}$ & -30.07 & & 4 & $2^{E}$ & 1 & -30.45 \\
$3^{+}$ & -27.03 & & 2 & $3^{E}$ & 0 & -27.48 \\
$4^{+}$ & -25.86 & & 4 & $3^{E}$ & 1 & -25.91 \\
\hline
\end{tabular}

corresponding energy levels for a $J \leq 5$ total angular momemtum. The negligible differences in energy with those reported calculations show the capabilities of the present approach. In addition to this, the use of symmetry adapted vibrational basis set and the procedure to assign the corresponding symmetry character to the rovibrational $J>0$ energy levels enables a complete description of the spectra of these kind of systems. The obtained results are as good as those previously reported for the Ar trimer. For $\mathrm{Ne}_{3}$ the contribution from Coriolis distortion terms, found relevant in a previous investigation for the much lighter system $\mathrm{H}_{3}^{+}$, does not seem to be important.

\section{Authors contributions}

All the authors were involved in the preparation of the manuscript. All the authors have read and approved the final manuscript.

\section{Acknowledgments}

This work has been funded by the Spanish MINECO with Grants Nos. FIS2014-51933-P, FIS2017-83157-P, FIS201452172-C2 and FIS2017-83473-C2.

\section{References}

1. N.J. Wright, J.M. Hutson, J. Chem. Phys. 110, 902 (1999)

2. P.N. Roy, J. Chem. Phys. 119, 5437 (2003)

3. S.W. Rick, D.L. Leitner, J.D. Doll, D.L. Freeman, D.D. Frantz, J. Chem. Phys. 95, 3506 (1991)

4. D.M. Leitner, J.D. Doll, R.M. Whitnell, J. Chem. Phys. 94, 6644 (1991)

5. D. Blume, C.H. Greene, B.D. Esry, J. Chem. Phys. 110 , $2145(2000)$
6. C. Chakravarty, R.J. Hinde, D.M. Leitner, D.J. Wales, Phys. Rev. E 56, 363 (1997)

7. T.R. Horn, R.B. Gerber, J.J. Valentini, M.A. Rater, J. Chem. Phys. 94, 6728 (1991)

8. A.R. Cooper, S. Jain, J.M. Hutson, J. Chem. Phys. 98, 2160 (1993)

9. A. Ernesti, J.M. Hutson, J. Chem. Phys. 103, 3386 (1995)

10. S. Garashchuk, J.C. Light, J. Chem. Phys. 114, 3929 (2001)

11. E.D. Belega, D.N. Trubnikov, L.L. Lohr, Phys. Rev. A 63, 043203 (2001)

12. L.L. Lohr, Int. J. Quant. Chem. 57, 708 (1996)

13. M. Salci, S.B. Levin, N. Elander, E. Yarevsky, J. Chem. Phys. 129, 134304 (2008)

14. B. Yang, W. Chen, B. Poirier, J. Chem. Phys. 135, 094306 (2011)

15. H. Suno, J. Chem. Phys. 135, 134312 (2011)

16. F. Karlický, B. Lepetit, R. Kalus, F.X. Gadéa, J. Chem. Phys. 126, 174305 (2007)

17. M. Márquez-Mijares, T. González-Lezana, O. Roncero, S. Miret-Artés, G. Delgado-Barrio, P. Villarreal, Chem. Phys. Lett. 460, 417 (2008)

18. M. Márquez-Mijares, R. Pérez de Tudela, T. GonzálezLezana, O. Roncero, S. Miret-Artes, G. Delgado-Barrio, P. Villarreal, I. Baccarelli, F.A. Gianturco, J. RubayoSoneira, J. Chem. Phys. 130, 154301 ( 11) (2009)

19. T. González-Lezana, S. Miret-Artés, G. Delgado-Barrio, P. Villarreal, J. Rubayo-Soneira, I. Baccarelli, F. Paesani, F.A. Gianturco, Comp. Phys. Comm. 145, 156 (2002)

20. T. González-Lezana, J. Rubayo-Soneira, S. Miret-Artés, F.A. Gianturco, G. Delgado-Barrio, P. Villarreal, Phys. Rev. Lett. 82, 1648 (1999)

21. T. González-Lezana, J. Rubayo-Soneira, S. Miret-Artés, F.A. Gianturco, G. Delgado-Barrio, P. Villarreal, J. Chem. Phys. 110, 9000 (1999)

22. I. Baccarelli, F.A. Gianturco, T. González-Lezana, G. Delgado-Barrio, S. Miret-Artés, P. Villarreal, J. Chem. Phys. 122, 084313 (2005)

23. I. Baccarelli, F.A. Gianturco, T. González-Lezana, G. Delgado-Barrio, S. Miret-Artés, P. Villarreal, J. Chem. Phys. 122, 144319 (2005)

24. I. Baccarelli, F.A. Gianturco, T. González-Lezana, G. Delgado-Barrio, S. Miret-Artés, P. Villarreal, Phys. Rep. 452, 1 (2007)

25. I. Baccarelli, F.A. Gianturco, T. González-Lezana, G. Delgado-Barrio, S. Miret-Artés, P. Villarreal, J. Phys. Chem. A 110, 5487 (2006)

26. T. González-Lezana, D. López, S. Miret-Artés, F.A. Gianturco, G. Delgado-Barrio, P. Villarreal, Chem. Phys. Lett. 335, 105 (2001)

27. M. Márquez-Mijares, O. Roncero, P. Villarreal, T. González-Lezana, Few-Body Systems 59, 14 (2018)

28. H.W. Kroto, in Molecular Rotation Spectra (Dover Publications, New York, 1992)

29. G.W. King, R.M. Hainer, P.C. Cross, J. Chem. Phys. 11, 27 (1942)

30. H.H. Nielsen, Rev. Mod. Phys 23, 90 (1951)

31. B.S. Ray, Z. Phys. 78, 74 (1932)

32. R.A. Aziz, M. Slaman, Chem. Phys. 130, 187 (1989) 